\title{
Psoriatic arthritis comorbidity index: development and validation of a new specific tool for classifying prognostic comorbidity in psoriasis and psoriatic arthritis patients
}

\author{
Y. E1 Miedany ${ }^{1,2 *}$, M. El Gaafary ${ }^{3}$, S. Youssef ${ }^{2}$, Sami Bahlas ${ }^{4}$ and M. Hegazi $^{5}$ \\ ${ }^{1}$ Rheumatology, Darent Valley Hospital, Dartford, United Kingdom \\ ${ }^{2}$ Rheumatology \& Rehab, Ain Shams University, Cairo, Egypt \\ ${ }^{3}$ Community and Public Health, Ain Shams University, Cairo, Egypt \\ ${ }^{4}$ Rheumatology, King Abdel Aziz University, Jeddah, Saudi Arabia; 5 Medicine, Al Adan Hospital, Kuwait
}

\begin{abstract}
Objective: 1. identify comorbidities with greatest impact on PsA patients' health status. 2.develop and validate a prospectively applicable comorbidity index for classifying PsA patients according to their comorbid conditions.

Methods: This was a retrospective multicenter cohort analysis of PsA patients in a rheumatology clinical registry, assessing the effect of different comorbidities measured at patients' visits over 10-years period. Outcomes of interest included functional ability, quality of life, medications induced complications, hospitalization/ death. A weighted index that was developed in a cohort of 1707 PsA patients. Internal and external validation were carried out.

Results: PsA patients who had higher incidence of comorbid condition and were at high risk of hospitalization were men, with older age at disease onset, high BMI ( $\mathrm{p}<0.05$ ). Multivariate regression analysis identified 29 comorbidities. A comorbidity index weighted according to the regression coefficient of the variables was developed (the score range: $0-37$ ). A cut off point of 8 was associated with a sensitivity of $97.5 \%$ and a specificity of $87 \%$. The developed PsACI correlated significantly with the 4 tested comorbidity indices: Charlson comorbidity index, Functional comorbidity index, Rheumatic Diseases comorbidity index, and Multimorbidity index at 1-, 3-, 5- and 10-years. Similar significant correlation was seen on external validation assessment.

Conclusion: The PsA-comorbidity index is a valid method for estimating comorbidity risk in PsA patients. It enables the clinicians to include comorbidities assessment and management in their practice. It can be used to predict resource utilization, identify targets for reducing costs, by prospectively identifying PsA patients at high risk.
\end{abstract}

\section{Introduction}

Rheumatic diseases are chronic progressive conditions which may result in significant physical disability and, in several cases, accelerated mortality [1-3]. While the primary disease accounts for much of the heightened death risk and the majority of morbidity, other factors such as comorbid conditions play a major contributing role $[4,5]$.

A widely accepted definition of comorbidity is "the existence or occurrence of any distinct additional entity during the clinical course of a patient who has the index disease under study" [6]. These additional entities are categorized according to the comorbidity 'three Cs' classification scheme: causality, complication, and coincidence. Causality is when an inflammatory disease like rheumatoid (RA), psoriatic arthritis (PsA) or systemic lupus erythematosus (SLE) is followed by patho-physiologically linked abnormalities, such as cardiovascular disorders; complications are comorbidities therapeutically linked to illnesses such as diabetes mellitus or osteoporosis (glucocorticoid-induced) or peptic ulcer (NSAIDinduced). Coincidence means that comorbidities occur independently and are unrelated to the index disease (for example, inflammatory arthritis and appendicitis) $[7,8]$. Separate patterns of comorbidity have been identified in patients with rheumatic diseases whether they have inflammatory arthritic conditions (such as RA, PsA, and SLE); or those with non-inflammatory rheumatic disorders such as fibromyalgia or osteoarthritis [9]. As these comorbidities contribute to increased early mortality, affect disease activity, response to treatments, and generate costs in these populations, they should be considered when managing patients with inflammatory arthritic conditions [10-12]. Furthermore, medications used to manage the underlying inflammatory condition, including disease- modifying antirheumatic drugs (whether synthetic (sDMARD), or biologic therapy (bDMARD), corticosteroids, and NSAID, may also increase or, conversely, decrease the likelihood of comorbidities.

People with psoriasis ( $\mathrm{PsO}$ ) and PsA were reported to have an elevated risk of developing comorbidities. A recent review noted that over half of the PsA patients have more than one comorbidity; which had a significant negative impact on their functional ability as well as quality of life [13]. There is currently no standardized, and validated instrument for recording and collecting comorbidity data in patients with $\mathrm{PsO}$ and PsA, which is relevant to contemporary and routine rheumatology practice.

Correspondence to: Y. El Miedany, Rheumatology, Darent Valley Hospital, Dartford, United Kingdom, E-mail: drelmiedany@rheumatology4u.com

Received: March 14, 2017; Accepted: April 10, 2017; Published: April 13, 2017 
El Miedany Y (2017) Psoriatic arthritis comorbidity index: development and validation of a new specific tool for classifying prognostic comorbidity in psoriasis and psoriatic arthritis patients

The purpose of the study is to identify comorbidities with greatest impact on PsO and PsA patients' health status. Also, to develop and validate a prospectively applicable comorbidity index for classifying these patients according to their comorbid conditions which might alter their risk of hospitalization and mortality.

\section{Methods}

\section{Study Design}

This was a retrospective multicenter cohort analysis of psoriatic patients in a rheumatology clinical registry assessing the effect of different comorbidities measured at patients' visits over 10-years period, on predicting future death and physical disability.

\section{Ethical Considerations}

Local ethical and methodological protocols for the study approval were followed. All patients included in this work signed an informed consent according to the Helsinki declaration.

\section{Patients}

\section{Recruitment}

Based on "psoriasis and psoriatic arthritis" referral pathway, psoriatic patients were referred to a specialized PsA clinic [14]. Arthritis was confirmed both clinically and radiologically using US (Mylab 25 esaote, Italy). MRI scan of the affected joint was carried out if further confirmation was required. Lab tests included: full blood count, chemistry, inflammatory markers (ESR and CRP), HLA-B27, thyroid function tests, bone profile, hepatitis markers, Rheumatoid factor, anti-nuclear antibodies (ANA), extractable nuclear antigen (ENA) and anti-CCP. X-ray of the affected joints was also carried out. Inclusion criteria: Adult patients, more than 18 years old, who have been suffering from PsA. All patients fulfilled the Classification criteria for Psoriatic Arthritis (CASPAR) criteria [15] and eligible for SDMARD or bDMARD therapies were included in this work. All the patients had chronic plaque psoriasis and had persistent pain involving any finger and/or toe for $>6$ weeks, or remittent pain involving any finger and/or toe for 3 months.

\section{Exclusion criteria}

1. Patients with history of RA, urogenital, intestinal or other forms of infection.

2. Patients taking oral steroids for non-arthritic/ other medical causes.

3. Patients with past history of cancer or hepatitis, HIV or any other contraindication to DMARDs therapy.

\section{Data Collection}

Prior to baseline examination in the clinic and every follow-up appointment, each patient completed a patient reported outcome measures (PROMs) questionnaire [16]. This is a self- completed questionnaire evaluating the disease activity parameters, functional ability as well as quality of life. To minimize the potential of missing data, patients who missed their appointment were contacted a new follow up appointment was set up. Patients who missed 2 consecutive appointments, were excluded from the work and statistical analysis.

Body mass index (BMI) was calculated from measures taken during the patient's outpatient appointments and categorized per WHO criteria as 'normal', 'overweight' and 'obese'. Smoking status was defined.
Disease Activity measures: Disease Activity for Psoriatic Arthritis (DAPSA) [17] was used as composite measure to assess the PsA disease activity. It encompasses 68 tender and 66 swollen joints count, scores for pain and patient global assessment a well as C-reactive protein level. The total score is a simple sum of the scores. Score $<4$ was considered as remission, in the range of $>4-<14$ low disease activity, $>14-<28$ moderate disease activity; whereas $>28$ reflect high disease activity. Skin affection was assessed using both they included Body Surface Area (BSA) [18], and Psoriasis Area and Severity Index (PASI) [19]. PASI score $<1$ was considered in remission, score $<10$ was considered as mild, 10-20 moderate whereas PASI score $>20$ was considered as severe. Enthesitis was assessed using the Maastricht Ankylosing Spondylitis Entheses Score (MASES) [20]. The assessment for spinal involvement was carried out using ASDAS [21]; whereas nail assessment was carried out using Nail Psoriasis Severity Index (NAPSI) [22]. Dactylitis was recorded as present or absent.

\section{Treatment protocol}

All the patients were treated according to a study protocol based on approved international guidelines [23]. Unless there was a contraindication, the patients with inflammatory PsA started their sDMARDs therapy once the diagnosis was confirmed. One off intramuscular bridge steroid injection was considered on starting sDMARD therapy as a bridge therapy. At 6-month from starting the $\operatorname{SDMARD}(\mathrm{s})$ therapy, bDMARD therapy, in addition to sDMARD medication, was commenced for those whose disease remained highly active, whereas switching to, or adding, sDMARD therapy was considered for those whose disease activity remained in the mild to moderate range. Switching bDMARD therapy was considered if the patient sustained untoward side effects, or showed no significant response in their DAPSA and PASI scores. The patients had the option of contacting the advice line and urgent assessments in the clinic were arranged whenever indicated. Follow up and database recording: Using "Electronic Outcome Measures for Inflammatory arthritis and spondylo-Arthritis/ EROMIA) [24], each patient's visit data were recorded.

\section{Comorbidity Assessment}

Screening for comorbidities was initially carried out as part of the PROMs self-administered questionnaire [16]. Participants were considered to have one or more comorbidity if they answered 'yes' to the following sequence of questions: 'Have you been informed or diagnosed to have any of these conditions?' This was verified by checking the patients' notes, lab as well as radiology outcome. The ICD10 record was checked and updated whenever appropriate. In case of death, cause of death was recorded. Similarly, the cause of hospitalization was recorded. Monitoring of comorbidities and risk factors: CV disease: a patient was considered as optimally monitored if all measurable risk factors for $\mathrm{CV}$ disease (BP, glycaemia, cholesterol, creatinine) were monitored at least once during the previous year. Infections: a patient was considered as optimally monitored (a) if dental examination had been conducted in the prior year; (b) for patients aged $>65$ years or receiving bDMARDs, they were considered as optimally vaccinated if they had received an influenza vaccination within the past 12 months and a pneumococcal vaccination within the past 5 years and (c) for patients ever exposed to bDMARD, they were considered as optimally screened for viral hepatitis (HBV and HCV) if they had ever been screened. Cancer: for each cancer, optimal monitoring was determined only for the population at risk (depending on the gender and age), according to each cancer screening recommendation. For breast cancer, individuals at risk were (a) women older than 50 years with 
E1 Miedany Y (2017) Psoriatic arthritis comorbidity index: development and validation of a new specific tool for classifying prognostic comorbidity in psoriasis and psoriatic arthritis patients

no history of breast cancer and (b) women of all ages with no personal history of breast cancer but with a family history of breast cancer; both groups were considered as optimally monitored if they had received a mammogram during the past 2 years. For cervix cancer screening, population at risk were women of all ages with no past history of cervix cancer; they were considered as optimally monitored if they had received a cervical smear test within the past 3 years. For colon cancer, optimal monitoring was dependent on the presence of risk factors: all patients older than 50 years were considered as optimally monitored if they had been tested for faecal occult blood at least once during the past 2-years; however, patients with at least one risk factor for colorectal cancer (a history of IBD, a family history of colon cancer or a family history of adenomatous polyposis) were only considered as optimally monitored if they had undergone a colonoscopy at least once. For skin cancer, the individuals at risk were patients with $>40$ naevi or who were ever exposed to biological DMARDs; they were considered as optimally monitored if they had visited a dermatologist within the last 12 months. Outcome of interest: The PsACI was assessed for impact on 4 outcomes: Functional ability, quality of life, medications induced complications, hospitalization and death. Linear regression was carried out using functional disability as the dependent variable.

\section{Validation and comparative assessment}

Content Validity refers to the extent to which a measure covers all facets of a given construct. The was carried out by assessment of the relative risk estimates of the proportional regression model to predict mortality using clinical data as well as the outcomes of interest.

Criterion validity is the extent to which the new index correlates with an existing one with the same construct. The developed comorbidity index was assessed in correlation with the Charlson comorbidity index [25], Multimorbidity index [26], rheumatic diseases comorbidity index (RDCI) [27] as well as functional disability comorbidity index [28].

Predictive validity refers to the degree the new index predicts functional disability and quality of life as well as hospitalization/ death in the future. The relation of developed comorbidity index was assessing in comparison with HRQoL (functional disability and quality of life) at year 3, 5 and 10 using linear regression and compared predicted versus observed values.

External validation of the developed PsACI was tested in 452 PsA patients included in another cross sectional observational study.

\section{Statistical Analysis}

Burden of comorbidities was described as frequency and $95 \%$ confidence intervals of different comorbidities among the PsO and PsA patients, cohort. Univariate analysis assessing the relationship between different comorbidities and disease parameters, functional disability, quality of life, medication induced complications and hospitalization / death was conducted using student t- test and chi-squared test for continuous and categorical data respectively. Along the 10-years follow up emerging comorbidities were recorded in the patient file and included in the current study database.

\section{Development of the comorbidity index}

Cox regression analysis was performed to identify the contribution of each of the previously mentioned comorbidities and disease parameters in survival. Regression coefficient of each of those variables was rounded to the nearest 0.5 number to obtain the weight of each of those predictors in the form of a score. The assigned score was multiplied by 1 if the comorbid condition or the disease parameter was present and by 0 if not. Summing up all the assigned score for different comorbidities and disease parameters, a total comorbidity score is calculated for each patient. The proposed comorbidity score was internally validated using ROC curve. Different coordinates of ROC curve were revised to select the cutoff point giving the highest sensitivity and specificity. Spearman correlation coefficient was calculated to test the relationship between the invented score and the 4 selected comorbidity indices. Critical $p$ value was always set at 0.5 . All statistical manipulation and analyses were performed using the 20th version of SPSS.

\section{Results}

In a cohort of 1707 Psoriatic arthritis patients, 851 (49.8\%) showed remission at the end of a 10- years follow. The 5-years survival rate was $99.4 \%$ and the 10 -years was $98.3 \%$. Psoriatic patients who were not hospitalized $(n=851)$ reported a lower age of onset of the disease, a female predilection and lower BMI at baseline data $(\mathrm{p}<0.05)$ than the PsA patients who were hospitalized because of comorbidity $(n=856)$. Prevalence of comorbidities: Over the 10-years follow up period, life time prevalence of different comorbidities was calculated; 29 comorbidities were identified. Table 1 shows the frequency and 95\% confidence interval of the comorbidities over the 10-years study period. There was significant variation $(\mathrm{p}<0.01)$ of the comorbidities prevalence at baseline and 3-years in contrast to 5- and 10-years disease duration. All the comorbidities were significantly associated with 10-years hospitalization/ mortality in the univariate analysis. Comparing the hospitalized to the non-hospitalized patients revealed significantly lower incidence of comorbidities in the non-hospitalized cohort $(\mathrm{p}<0.01)$. Comparing the 2 groups, binary regression analysis revealed the factors: male gender, life time cerebrovascular or cardiovascular diseases, diabetes/ metabolic syndrome, osteoporosis, evident fall risk, diabetes, infection, anxiety/depression, liver, renal, lung and GIT diseases as well as the disease severity score were significant independent factors affecting the 10-years outcome of the disease controlling for age of onset of the disease. Whether raw or categories, the identified comorbidities as well as the disease severity were significantly lower in the patient cohort who did not report medication associated complications $(\mathrm{p}<0.01)$.

\section{Correlation with Disease Severity}

Multivariate regression analysis identified Multidimensional Disease Severity score (including 5 parameters: DAPSA $>4$, PASI $>10$, Functional disability score $>2$, enthesitis $>5 / 13$, ESR $>30 \mathrm{~mm} / \mathrm{hr}$-CRP $>15 \mathrm{mg} / \mathrm{dl}$ ) as independent predictor for disease status associated with the 10-year death risk or hospitalization (Wald $\chi 2=9.2, p=0.002$, $\mathrm{OR}=24.6$ ). If the patient got 3 or more of these parameters, the disease is considered as severe. The regression model showed discriminating ability between patients who were hospitalized versus those who did not $(\mathrm{p}<0.001)$, as well as the patient cohort who sustained medication associated complications versus those who did not $(\mathrm{p}<0.001)$. The Comorbidity Index: Table 2 shows the beta-coefficients and p-values by linear regression analyses reflecting the association of each morbid condition with functional disability; as well as the assigned weights in accordance to the beta-coefficients. Regression coefficients were rounded to whole number for easier manipulation and use. The score was ranging between 0 and 37. The higher the score, the higher the probability of a psoriatic patient to get hospitalized. 
El Miedany Y (2017) Psoriatic arthritis comorbidity index: development and validation of a new specific tool for classifying prognostic comorbidity in psoriasis and psoriatic arthritis patients

Table 1. The frequency and $95 \%$ confidence interval of the comorbidities recorded over the 10 -years study period

\begin{tabular}{|c|c|c|c|c|c|c|c|c|c|c|c|c|}
\hline \multirow[t]{2}{*}{ Comorbidity } & \multicolumn{3}{|c|}{ Baseline } & \multicolumn{3}{|c|}{ At 3-years } & \multicolumn{3}{|c|}{ At 5-years } & \multicolumn{3}{|c|}{ At 10-years } \\
\hline & No & $\%$ & $95 \% \mathrm{CI}$ & No & $\%$ & $95 \% \mathrm{CI}$ & No & $\%$ & $95 \% \mathrm{CI}$ & No & $\%$ & $95 \% \mathrm{CI}$ \\
\hline Depression & 1065 & 62.4 & $60.1-64.2$ & 523 & 30.6 & $28.3-32.9$ & 287 & 16.8 & $14.3-18.9$ & 301 & 17.6 & $15.3-19.8$ \\
\hline Anxiety & 1204 & 59.3 & $57.2-61.5$ & 673 & 39.4 & $37.5-42.1$ & 432 & 19 & $17.1-21.2$ & 357 & 20.9 & 18.4- 20.2 \\
\hline Diabetes M & 296 & 17.3 & $15.2-19.5$ & 391 & 22.9 & $20.7-24.9$ & 523 & 30.6 & $28.5-32.7$ & 667 & 39.1 & $36.8-41.3$ \\
\hline Met Syn & 121 & 8.6 & $6.2-10.3$ & 149 & 10.6 & 8.4- 12.9 & 249 & 14.6 & $12.5-16.8$ & 261 & 15.3 & $13.2-17.6$ \\
\hline IHD & 124 & 8.8 & $6.6-10.9$ & 381 & 26.3 & $24.7-28.6$ & 667 & 39.1 & $37.3-41.2$ & 873 & 51.1 & 49.353 .6 \\
\hline MI & 3 & 0.2 & $0.1-0.3$ & 35 & 2.1 & $0.6-4.2$ & 466 & 27.3 & 24.9- 28.7 & 523 & 30.6 & $28.3-32.9$ \\
\hline Hypertension & 148 & 10.5 & $8.2-12.8$ & 183 & 13 & $11.1-15.1$ & 442 & 31.5 & $29.2-33.6$ & 533 & 38 & $36.1-40.3$ \\
\hline Hyperlipdemia & 222 & 13 & $11.2-15.3$ & 256 & 15 & $13.3-17.1$ & 667 & 39.1 & $37.4-41.8$ & 716 & 41.9 & $39.6-43.8$ \\
\hline Arrhythmia & 49 & 3.5 & 3.3- 3.7 & 180 & 10.5 & $8.2-12.6$ & 248 & 14.5 & $12.2-16.8$ & 357 & 20.9 & $18.3-23.1$ \\
\hline CVD & 4 & 0.3 & $0.1-0.5$ & 51 & 3.6 & $1.7-5.5$ & 294 & 17.2 & $15.2-19.3$ & 533 & 31.2 & $29.2-33.5$ \\
\hline PVD & 3 & 0.2 & $0.0-0.4$ & 54 & 3.8 & 1.9- 5.4 & 259 & 15.2 & $13.4-17.2$ & 391 & 22.9 & $20.2-24.8$ \\
\hline Osteoporosis & 202 & 14.4 & $12.3-16.5$ & 497 & 24.5 & $22.6-26.4$ & 717 & 51.1 & $49.1-53.3$ & 404 & 28.8 & $26.7-30.8$ \\
\hline Fracture & 5 & 0.3 & $0.1-0.5$ & 27 & 1.3 & $0.3-3.1$ & 124 & 8.8 & $6.4-10.6$ & 582 & 34.1 & $32.4-36.3$ \\
\hline Fall & 62 & 4.4 & $2.3-6.6$ & 334 & 23.8 & $21.2-25.9$ & 408 & 23.9 & $21.7-25.6$ & 523 & 30.6 & 28.4- 32.8 \\
\hline Liver disease & 20 & 1.2 & $0.2-3.8$ & 53 & 3.1 & 1.3- 5.4 & 137 & 8 & $6.1-10.1$ & 207 & 12.1 & $10.1-14.2$ \\
\hline Renal disease & 30 & 1.8 & $0.7-4.1$ & 35 & 2.1 & $0.2-4.2$ & 112 & 6.6 & $4.3-8.9$ & 198 & 11.6 & $9.5-13.7$ \\
\hline Pulmonary & 66 & 4.5 & $1.3-6.7$ & 111 & 6.5 & $4.3-8.7$ & 181 & 10.6 & 8.4- 12 & 293 & 17.1 & $15.1-19.4$ \\
\hline GIT disease & 20 & 1.2 & $0.3-3.8$ & 294 & 17.2 & $15.1-19.5$ & 391 & 22.9 & $20.2-25.1$ & 533 & 31.2 & $29.0-33.4$ \\
\hline Endocrine & 32 & 1.9 & $0.6-4.1$ & 55 & 3.2 & $1.1-5.3$ & 64 & 4.5 & 2.4- 6.7 & 114 & 7.8 & 5.6- 9.9 \\
\hline Periodontitis & 3 & 0.2 & $0.0-0.4$ & 5 & 0.4 & $0.1-0.6$ & 49 & 3.5 & 1.3- 5.7 & 54 & 3.8 & $1.7-5.9$ \\
\hline Smoking & 201 & 14.3 & $12.2-16.5$ & 294 & 17.2 & $15.1-19.4$ & 258 & 15.1 & 13. & 208 & 12.2 & $10.1-14.3$ \\
\hline Infection & 35 & 2.1 & $0.4-4.1$ & 112 & 6.6 & $4.5-8.8$ & 202 & 14.4 & $12.2-16.7$ & 500 & 29.3 & 27.4- 31.1 \\
\hline Vasculitis & 10 & 0.8 & $0.2-2.2$ & 36 & 2.2 & $0.8-4.1$ & 134 & 9.7 & $7.5-11.4$ & 203 & 14.4 & $12.3-16.6$ \\
\hline Osteoarthritis & 66 & 4.6 & $3.7-5.6$ & 131 & 9.1 & $7.0-11.2$ & 261 & 15.4 & 13.6- 17.2 & 669 & 39.2 & 37.4- 41.1 \\
\hline Fibromyalgia & 36 & 2.2 & $1.1-3.2$ & 111 & 6.5 & $5.3-7.7$ & 149 & 10.6 & $8.2-12.4$ & 203 & 14.4 & $12.5-16.7$ \\
\hline Amyloidosis & 0.0 & 0.0 & 0.0 & 0.0 & 0.0 & 0.0 & 2 & 0.1 & $0.0-0.3$ & 5 & 0.4 & $0.2-0.6$ \\
\hline Eye inflame/uveitis & 38 & 2.3 & $0.5-4.6$ & 69 & 4.1 & $2.2-6.1$ & 114 & 6.7 & $5.6-7.7$ & 201 & 14.3 & $12.6-16.1$ \\
\hline Tumour & 2 & 0.1 & $0.0-2.1$ & 14 & 0.8 & $0.2-2.4$ & 27 & 1.3 & $0.2-3.6$ & 112 & 6.6 & $4.2-8.8$ \\
\hline
\end{tabular}

Met Syn: Metabolic Syndrome, IHD: Ischemic heart disease, MI: Myocardial infarction, CVD: cerebrovascular disease, PVD: peripheral vascular disease, Diabetes M: Diabetes Mellitus

\section{Validation}

Multivariate linear regression analysis for functional disability score prediction using the developed PsACI adjusted for age and gender, showed significant correlation at 1-, 3-, 5- and 10-years (Table 3). Similar significant correlation was seen with Quality of life ( $\mathrm{p}<$ 0.001). Table 4 shows the correlation of the developed PsACI with the 4 tested comorbidity Indices at 1, 3, 5 and 10-years. There were variable correlation levels reflecting the variation of the disease duration in the different comorbidity indices studies. Similar significant correlation was seen on external validation assessment (Table 5). The ROC curve displaying the discriminating ability of the developed PsACI is shown in (Figure 1). This depicted an AUC (Area Under Curve) of 0.985 (95\% CI 0. 984-0.991). Different coordinate's points yield different sensitivity and specificity. A cut off point of 8 was associated with a sensitivity of $97.5 \%$ and a specificity of $87 \%$.

\section{Discussion}

Psoriasis is manifested as a systemic disorder accompanied by inflammatory consequences and comorbidities with negative impact on the patient, rather than a simple cutaneous disease. Results of this study revealed that patients with psoriatic arthritis have an increased comorbidity risk. These findings are in agreement with earlier published studies carried out to determine the risk of mortality in patients with psoriasis when compared to the general population and that PsA, by itself, is an independent risk factor for comorbidities particularly CVD [29]. Another Swedish study reported that patients with mild psoriasis did not have high mortality risk whereas severe psoriasis patients had an increased cardiovascular mortality risk [30]. This was supported by the data revealed from an independent study [31] which demonstrated that psoriasis patients may have decreased longevity.

The decrease in longevity may be as much as 20 -years in patients whose psoriasis begins before 25years of age. Data also supported that the age of mortality may be linked to the psoriasis age of onset. Among the 29 comorbidities reported in this work, CVD and diabetes were the most prevalent comorbidities which had the greatest impact on a patient's life. Recognizing and addressing comorbidities in PsA patients is critical to timely, safely and effectively treating them, as these comorbidities have implications both on the patient as well as therapy choices. Providing comprehensive care including comorbidity assessment for psoriatic patients is therefore highly recommended. Focusing treatment plans on just a single aspect of the disease spectrum risks ignoring issues that may have significant impact on patient function and quality of life.

In 1999, the American Heart Association and American Diabetes Association published a report on prevention of CVD in patients with diabetes mellitus [32]. Since that time, the cardiovascular risk in diabetic patients has been significantly lower than in earlier decades. Given the increased CVD and diabetes risk in PsA; screening, identification of risk factors and risk management became highly recommended. This was endorsed by the European League Against Rheumatism (EULAR) recommendations [33]. The advantage of comorbidity indices is that by reducing all coexistent disorders and their severity into a single numeric score, makes a comparison of this comorbidity score between patients possible. 
El Miedany Y (2017) Psoriatic arthritis comorbidity index: development and validation of a new specific tool for classifying prognostic comorbidity in psoriasis and psoriatic arthritis patients

Table 2. The beta-coefficients and p-values of the different comorbidities identified by linear regression analyses and its assigned weights in accordance to the beta-coefficients.

\begin{tabular}{|c|c|c|c|c|}
\hline \multirow{2}{*}{$\begin{array}{c}\text { PsACI } \\
29 \text { Comorbidities }\end{array}$} & \multicolumn{2}{|c|}{ Regression Coefficient } & \multirow{2}{*}{$\mathrm{P}$-value } & \multirow{2}{*}{$\begin{array}{c}\text { Assigned } \\
\text { Score } \\
0-37\end{array}$} \\
\hline & B & Std. Error & & \\
\hline Disease Severity score $>3$ & 5.12 & 0.26 & 0.001 & 5 \\
\hline Metabolic Syndrome & 3.26 & 0.29 & 0.001 & 3 \\
\hline Myocardial Infarction & 2.27 & 0.31 & 0.001 & 2 \\
\hline Ischemic Heart Disease & 2.21 & 0.28 & 0.001 & 2 \\
\hline Depression & 1.99 & 0.23 & 0.001 & 2 \\
\hline Diabetes Mellitus & 2.4 & 0.22 & 0.001 & 2 \\
\hline Fracture & 2.13 & 0.27 & 0.001 & 2 \\
\hline Hypertension & 1.21 & 0.22 & 0.001 & 1 \\
\hline Hyperlipidaemia & 1.23 & 0.21 & 0.002 & 1 \\
\hline Arrhythmia & 1.25 & 0.23 & 0.001 & 1 \\
\hline CVD & 1.23 & 0.26 & 0.001 & 1 \\
\hline PVD & 1.41 & 0.27 & 0.005 & 1 \\
\hline Fall & 1.14 & 0.25 & 0.003 & 1 \\
\hline Liver disease & 1.24 & 0.27 & 0.006 & 1 \\
\hline Pulmonary disease & 1.26 & 0.22 & 0.005 & 1 \\
\hline Endocrine & 1.32 & 0.28 & 0.001 & 1 \\
\hline GIT & 1.41 & 0.29 & 0.002 & 1 \\
\hline Renal & 1.3 & 0.21 & 0.005 & 1 \\
\hline Tumour & 1.14 & 0.28 & 0.004 & 1 \\
\hline Infection & 1.2 & 0.23 & 0.004 & 1 \\
\hline Anxiety & 1.11 & 0.26 & 0.003 & 1 \\
\hline Smoking & 1.41 & 0.28 & 0.001 & 1 \\
\hline Osteoporosis & 1.32 & 0.21 & 0.003 & 1 \\
\hline Periodontitis & 0.57 & 0.21 & 0.001 & 0.5 \\
\hline Osteoarthritis & 0.61 & 0.24 & 0.001 & 0.5 \\
\hline Fibromyalgia & 0.67 & 0.26 & 0.001 & 0.5 \\
\hline $\begin{array}{c}\text { Eye inflammation/ } \\
\text { uveitis }\end{array}$ & 0.63 & 0.23 & 0.001 & 0.5 \\
\hline Vasculitis & 0.64 & 0.22 & 0.001 & 0.5 \\
\hline Amyloidosis & 0.55 & 0.25 & 0.001 & 0.5 \\
\hline
\end{tabular}

CVD: Cerebrovascular Disease, PVD: Peripheral vascular disease

Table 3. Multivariate Linear Regression Analysis for Functional Disability Score Prediction using Comorbidities Adjusted for Age and Gender

\begin{tabular}{|c|c|c|c|c|}
\hline & 1-year & 3-year & 5-year & 10 -year \\
\hline F-value & 0.663 & 0.773 & 0.821 & 0.868 \\
\hline p-value & $<0.001$ & $<0.001$ & $<0.001$ & $<0.001$ \\
\hline $\mathrm{R}^{2}$ & 0.725 & 0.773 & 0.847 & 0.872 \\
\hline Adjust $\mathrm{R}^{2}$ & 0.723 & 0.771 & 0.846 & 0.871 \\
\hline
\end{tabular}

Table 4. Correlation of the PsACI score with Comorbidity Indices at 1, 3, 5 and 10-years

\begin{tabular}{|c|c|c|c|c|}
\hline $\begin{array}{c}\text { Comorbidity } \\
\text { Index }\end{array}$ & PsACI at 1-year & PsACI at 3-years & PsACI at 5-years & $\begin{array}{c}\text { PsACI at } \\
10 \text {-years }\end{array}$ \\
\hline CCI & $0.325^{*}$ & $0.436^{*}$ & $0.574^{*}$ & $0.791^{*}$ \\
\hline FCI & $0.762^{*}$ & $0.566^{*}$ & $0.743^{*}$ & $0.895^{*}$ \\
\hline RDCI & $0.863^{*}$ & $0.789^{*}$ & $0.886^{*}$ & $0.916^{*}$ \\
\hline MMI & $0.747^{*}$ & $0.732^{*}$ & $0.786^{*}$ & $0.903^{*}$ \\
\hline
\end{tabular}

CCI: Charlson comorbidity index, FCI: Functional comorbidity index, RDCI: Rheumatic Diseases comorbidity index, MMI: Multimorbidity index. ${ }^{*} \mathrm{p}<0.01$

Few comorbidity indices are already available and have been assessed in previous studies. The Charles comorbidity Index (CCI) was the outcome of a study published in 1987 [15], based on the mortality rates of 607 patients admitted for a period of 1-month under the internal medicine care. Seventeen diseases were encompassed in that index, with different weights. They were identified and weighted according to the strength of their association with 1-year mortality. Connective tissue diseases were included as one of the disorders with adjust risk of 1 . Later on, using administrative data, Elixhauser et al. [32] identified 30 comorbidities (the 17 from CCI +13 new ones) which had high impact on short-term outcomes in acute hospital patients. Calculation of total score was based on giving 1 point per comorbidity; adding all items would give the Elixhauser comorbidity score (ECS). Both these indices won't be the best applicable model for assessing PsA patients. While the CCI was developed to predict death in a sample of hospitalized patients, it has been widely implemented outside its originally proposed scope. Similarly, the ECS was developed using a cohort of hospitalized patients to predict length of hospital charges, hospital stay, and the risk of in-hospital death. Like the CCI, the ECS has also been used in situations beyond its envisioned scope. Furthermore, in the current PsA management context, both Charlson and Elixhauser indices can be considered as outdated. Most of the PsA patients (number unknown) included in the CCI most likely have not, at all, received methotrexate therapy and had very long disease duration, whereas most of the patients included in ECS most likely have missed the biologic therapy era. Furthermore, in ECS, a comorbidity count was used.

Use of a comorbidity count is discouraged for 2 reasons; firstly, a wide variability in predictive ability is expected; and secondly, comorbidity counts are expected to vary in the number and types of comorbidities encompassed. On another front, The Rheumatic Disease Comorbidity Index (RDCI) was developed from self-report questionnaires from patients with RA, systemic lupus erythematosus, fibromyalgia or osteoarthritis. [33]. No PsA patients were included.

Table 5. External validation: correlation of the PsACI with functional disability, quality of life as well as the comparator comorbidity indices.

\begin{tabular}{|c|c|c|}
\hline & Spearman Correlation Coefficient & P-value \\
\hline Functional Disability & 0.892 & $<0.001$ \\
\hline Quality of life & 0.863 & $<0.001$ \\
\hline Multimorbidity Index & 0.931 & $<0.001$ \\
\hline Rheumatic Disease Comorbidity Index & 0.929 & $<0.001$ \\
\hline Functional comorbidity index & 0.887 & $<0.001$ \\
\hline Charlson Comorbidity Index & 0.746 & $<0.001$ \\
\hline
\end{tabular}

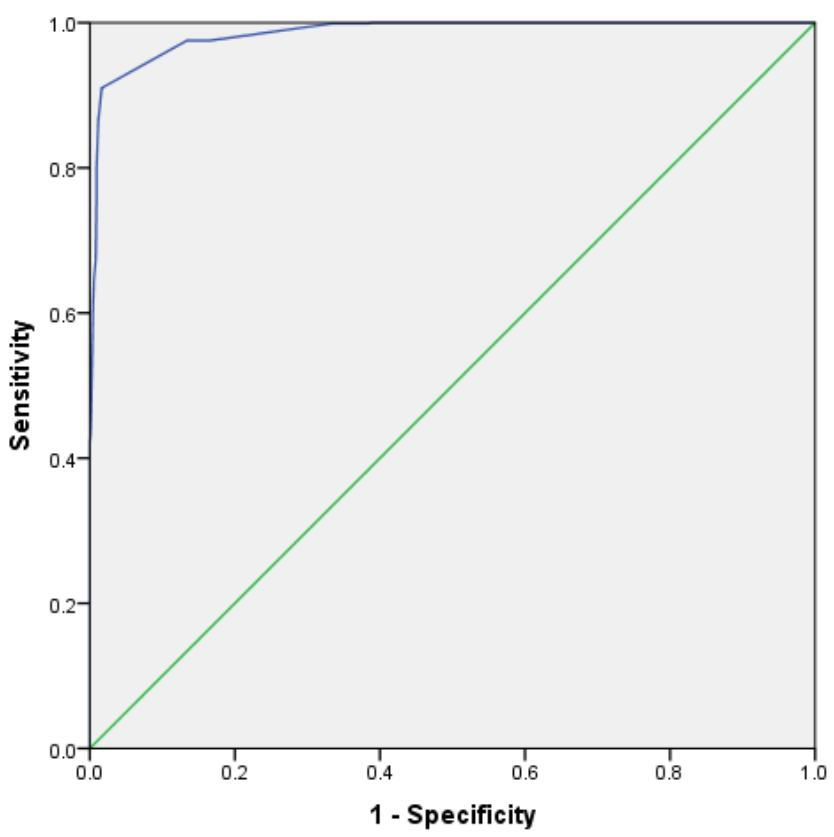

Figure 1. ROC curve displaying the discriminating ability of the PsACI 
El Miedany Y (2017) Psoriatic arthritis comorbidity index: development and validation of a new specific tool for classifying prognostic comorbidity in psoriasis and psoriatic arthritis patients

Twenty-two comorbid illnesses were weighed for impact on 6 outcomes: work disability, Health Assessment Questionnaire (HAQ) functional disability, social security disability, direct medical costs, hospitalization, and death. The final score includes 11 comorbid conditions. However, the RCDI index had fixed baseline values for analysis, thus it removed the chronological component of comorbidity during the follow-up period. This reduces the predictive power of comorbidity indices [34]. Additionally, data were collected based on ICD-9-CM codes recorded from the outpatient appointments, which rely on the providers to maintain an accurate list of comorbid conditions. Lastly, all the patients included in the RDCI were males in contrast with most of the inflammatory arthritic conditions which is more prevalent in women [35]. This study presents a comorbidity index developed based on real life scenario of the current clinical practice. The PsA patients included both men and women who had short disease duration, and the patients were monitored for 10-years. Furthermore, the disease activity state for the first time was included as one of the risk factors.

Defining the relationships between comorbidities and $\mathrm{PsO} / \mathrm{PsA}$ disease activity may help identify subsets of PsA patients at high risk for comorbidities. Results revealed higher numbers of comorbidities reported in association with high PsA disease activity, duration and functional limitations. These findings are in agreement with an earlier study [34] which revealed that the number of comorbidities in 190 PsA patients was associated with functional ability as well as disease duration of 5-15 years. In other reviews $[35,36]$ psoriatic skin disease severity has been shown to be more likely associated with comorbidities. This was endorsed by the results of another work which revealed that the hazard ratio of mortality in psoriatic patients was increased, particularly in patients with severe psoriasis who are aged under 55 years. These results were in concordance with the outcomes of Gelfand et al study [37], where patients with very severe psoriasis were found to be associated with increased non-cardiovascular mortality. Furthermore, in agreement with this work, the study showed that data on comorbidity could be collected with questionnaires, as well as ICD-10.

Self-administered questionnaires could be a valid and reliable alternative approach to assess comorbidities, and a tool to be included in prospective studies. The self- administered comorbidity questionnaire (SCQ) was published by Sangha et al. [38] include questions for the patients to indicate whether they suffer, at the moment, from 12 additional medical illnesses identified by an expert panel based on the comorbidities collected on the CCI. The score ranges from 0 to 45 points. Results of this work revealed that it is possible to incorporate comorbidity assessment in the standard patient reported outcome measure questionnaire setting, this enables the treating health care professionals to link between the patients' comorbidity, the disease activity as well as their functional ability and quality of life scores.

In conclusion, psoriasis is a complex inflammatory disease that shares many immunological features with other complex disorders, such as CVD, depression, obesity, diabetes, and inflammatory arthritis. Comorbidities have been linked to disease severity, duration as well as activity. In parallel with the increase of the comorbidities number, there is a similar increase in the healthcare utilization and healthcare costs. Both the rheumatologists and dermatologists not only need to educate their patients of these risks, but also be proactive in their assessment and monitoring of these patients. Whilst knowledge of these risk factors is important in terms of the patients' general health, they may also have an impact on how these patients are managed [39].

\section{Competing Interest}

The authors have no relevant financial disclosures.

\section{Contributorship}

All authors were involved in drafting the article or revising it critically for important intellectual content, and all authors approved the final version to be submitted for publication. Dr. El Miedany had full access to all of the data in the study and Dr. El Gaafary carried out the data analysis.

\section{Acknowledgements}

We express thanks to all participants, our colleagues, research assistants and nurses for their cooperation and help to bring this research to its final conclusions.

\section{References}

1. Wolfe F, Mitchell DM, Sibley JT, Fries JF, Bloch DA, et al. (1994) The mortality of rheumatoid arthritis. Arthritis Rheum 37: 481-494. [Crossref]

2. Myasoedova E, Davis JM 3rd, Crowson CS, Gabriel SE (2010) Epidemiology of rheumatoid arthritis: rheumatoid arthritis and mortality. Curr Rheumatol Rep 12: 379385. [Crossref]

3. Sokka T, Krishnan E, Hakkinen A, Hannonen P (2003) Functional disability in rheumatoid arthritis patients compared with a community population in Finland. Arthritis Rheum 48:59-63. [Crossref]

4. Escalante A, del Rincón I (1999) How much disability in rheumatoid arthritis is explained by rheumatoid arthritis? Arthritis Rheum 42: 1712-1721. [Crossref]

5. Radner H, Smolen JS, Aletaha D (2010) Impact of comorbidity on physical function in patients with rheumatoid arthritis. Ann Rheum Dis 69: 536-541. [Crossref]

6. Feinstein AR (1970) THE PRE-THERAPEUTIC CLASSIFICATION OF COMORBIDITY IN CHRONIC DISEASE. J Chronic Dis 23: 455-468. [Crossref]

7. Schellevis FG, van Eijk JT, van den Lisdonk EH, vd Velden J, van Weel C (1994) Implementing guidelines in general practice. Evaluation of process and outcome of care in chronic diseases. Int J Qual Health Care 6: 257-266. [Crossref]

8. Schellevis FG, van der Velden J, van de Lisdonk E, van Eijk JT, van Weel C (1993) Comorbidity of chronic diseases in general practice. J Clin Epidemiol 46: 469-473. [Crossref]

9. Wolfe F, Michaud K, Li T, Katz RS (2010) Chronic conditions and health problems in rheumatic diseases: comparisons with RA, noninflammatory rheumatic disorders, SLE \& fibromyalgia. $J$ Rheumatol 37: 305-315. [Crossref]

10. Haraoui B, Liu PP, Papp KA (2012) Managing cardiovascular risk in patients with chronic inflammatory diseases. Clin Rheumatol 31: 585-594. [Crossref]

11. Gulliver W (2008) Long-term prognosis in patients with psoriasis. Br J Dermatol 159 Suppl 2: 2-9. [Crossref]

12. Kimball AB, Guérin A, Tsaneva M, Yu AP, Wu EQ, et al. (2011) Economic burden of comorbidities in patients with psoriasis is substantial. J Eur Acad Dermatol Venereol 25: 157-163. [Crossref]

13. Ogdie A, Schwartzman S, Husni ME (2015) Recognizing and managing comorbidities in psoriatic arthritis. Curr Opin Rheumatol 27: 118-126. [Crossref]

14. El Miedany Y, El Gaafary M, Youssef S, Ahmed I, Nasr A (2015) Tailored approach to early psoriatic arthritis patients: clinical and ultrasonographic predictors for structural joint damage. Clin Rheumatol 34: 307-313. [Crossref]

15. Taylor W, Gladman D, Helliwell P, Marchesoni A, Mease P, et al. (2006) Classification criteria for psoriatic arthritis: development of new criteria from a large international study. Arthritis Rheum 54: 2665-2673. [Crossref]

16. El Miedany Y, El Gaafary M, Youssef S, Palmer D (2010) Towards a multidimensional patient reported outcome measures assessment: development and validation of a questionnaire for patients with ankylosing spondylitis/spondyloarthritis. Joint Bone Spine 77: 575-581. [Crossref]

17. Schoels M, Aletaha D, Funovits J, Kavanaugh A, Baker D, et al. (2010) Application of the DAREA/DAPSA score for assessment of disease activity in psoriatic arthritis. Ann Rheum Dis 69: 1441-1447. [Crossref]

18. Ramsay B, Lawrence CM (1991) Measurement of involved surface area in patients with psoriasis. Br J Dermatol 124: 565-570. [Crossref]

19. Fredriksson T, Pettersson U (1978) Severe psoriasis--oral therapy with a new retinoid. Dermatologica 157: 238-244. [Crossref] 
El Miedany Y (2017) Psoriatic arthritis comorbidity index: development and validation of a new specific tool for classifying prognostic comorbidity in psoriasis and psoriatic arthritis patients

20. Heuft-Dorenbosch L, Spoorenberg A, van Tubergen A, Landewé R, van ver Tempel $\mathrm{H}$, et al. (2003) Assessment of enthesitis in ankylosing spondylitis. Ann Rheum Dis 62 . 127-132. [Crossref]

21. Machado P, Landewé R, Lie E, Kvien T, Braun J, et al. (2011) Ankylosing Spondylitis Disease Activity Score (ASDAS): defining cut-off values for disease activity states and improvement scores. Ann Rheum Dis 70: 47-53. [Crossref]

22. Rich P, Scher RK (2003) Nail Psoriasis Severity Index: a useful tool for evaluation of nail psoriasis. J Am Acad Dermatol 49: 206-212. [Crossref]

23. Gossec L, Smolen J, Ramiro S, de Wit M, Cutolo M, et al. (2016) European League Against Rheumatism (EULAR) recommendations for the management of psoriatic arthritis with pharmacological therapies: 2015 update. Ann Rheum Dis 75: 499-510. [Crossref]

24. Palmer D, El Miedany Y (2010) EROMIA in inflammatory arthritis: the next step in standard practice. Br J Nurs 19: 42-46. [Crossref]

25. Charlson ME, Pompei P, Ales KL, MacKenzie CR (1987) A new method of classifying prognostic comorbidity in longitudinal studies: development and validation. J Chronic Dis 40: 373-383. [Crossref]

26. Radner H, Yoshida K, Mjaavatten MD, Aletaha D, Frits M, et al. (2015) Development of a multimorbidity index: Impact on quality of life using a rheumatoid arthritis cohort. Semin Arthritis Rheum 45: 167-173. [Crossref]

27. Michaud K, Wolfe F (2007) Comorbidities in rheumatoid arthritis. Best Pract Res Clin Rheumatol 21: 885-906. [Crossref]

28. Groll DL, To T, Bombardier C, Wright JG (2005) The development of a comorbidity index with physical function as the outcome. J Clin Epidemiol 58: 595-602. [Crossref]

29. Polachek A, Touma Z, Anderson M, Eder L (2017) Risk of Cardiovascular Morbidity in Patients With Psoriatic Arthritis: A Meta-Analysis of Observational Studies. Arthritis Care Res (Hoboken) 69: 67-74. [Crossref]
30. Gelfand JM, Troxel AB, Lewis JD, Kurd SK, Shin DB, et al. (2007) The risk of mortality in patients with psoriasis: results from a population-based study. Arch Dermatol 143: 1493-1499. [Crossref]

31. Gulliver WP (2007) Comorbidities associated with the Newfoundland and Labrador founder population. Presented at: 21st World Congress of Dermatology, Buenos Aires, Argentina, 30 September-5 October, 2007.

32. Peters MJ, Nurmohamed MT (2013) Cardiovascular risk management in rheumatoid arthritis: are we still waiting for the first step? Arthritis Res Ther 15: 111. [Crossref]

33. Peters MJ, Symmons DP, McCarey D, Dijkmans BA, Nicola P, et al. (2010) EULAR evidence-based recommendations for cardiovascular risk management in patients with rheumatoid arthritis and other forms of inflammatory arthritis. Ann Rheum Dis 69: 325-331.

34. Tanner S, McFadden M, Clegg D, Walsh J (2014) Relationship Between Psoriatic Arthritis Severity, Duration, and Comorbidities. Athritis rheumatism 2014, abstract number 1570

35. Kiliç A, Cakmak S (2013) Psoriasis and comorbidities. EMJ Dermatol 1:78-85

36. Husni ME (2015) Comorbidities in Psoriatic Arthritis. Rheum Dis Clin North Am 41 : 677-698. [Crossref]

37. Gelfand JM, Troxel AB, Lewis JD, Kurd SK, Shin DB, et al. (2007) The risk of mortality in patients with psoriasis: results from a population-based study. Arch Dermatol 143: 1493-1499. [Crossref]

38. Sangha O, Stucki G, Liang M, Fossel AH, Katz JN (2003) The Self-Administered Comorbidity Questionnaire: a new method to assess comorbidity for clinical and health services research. Arthritis Rheum 49: 156-63. [Crossref]

39. Desai SS, Myles JD, Kaplan MJ (2012) Suboptimal cardiovascular risk factor identification and management in patients with rheumatoid arthritis: a cohort analysis. Arthritis Res Ther 14: R270. [Crossref]

Copyright: (C2017 El Miedany Y. This is an open-access article distributed under the terms of the Creative Commons Attribution License, which permits unrestricted use, distribution, and reproduction in any medium, provided the original author and source are credited. 